\title{
Do precursor polyp burdens help distinguish Lynch versus non-Lynch microsatellite unstable colorectal cancers?
}

\author{
Craig Messick, James M Church, Graham Casey, Matthew F Kalady* \\ From 13th Annual Meeting of the Collaborative Group of the Americas on Inherited Colorectal Cancer \\ Honolulu, Hawaii, USA. 16-17 October 2009
}

\section{Background}

Microsatellite instability (MSI) within colorectal cancers (CRC) may develop through inherited germline mutations in mismatch repair (MMR) genes (Lynch Syndrome) or sporadic epigenetic methylation of tumor suppressor or repair genes (methylator pathway). Although the molecular mechanisms in each pathway have been described, their associated precursor polyp burdens are not well-defined. This study analyzes precursor polyp burdens occurring within patients with MSI-H colorectal cancers associated with Lynch Syndrome (LS) or those with a methylator pathway cancer phenotype.

\section{Methods}

MSI-H CRCs were identified either from an inherited CRC registry or clinic-based frozen tissue bank. Patients with confirmed MMR germline defects were defined as LS. MSI-H tumors considered to be methylators were defined by previously determined CpG island methylator phenotype (CIMP). These two groups were also compared to MSS/CIMP-negative tumor controls. Corresponding patient demographics, all prior preoperative colonoscopy, operative, and pathology reports for polyp characteristics were reviewed.

\section{Results}

114 patients were included: 29 LS, 22 Methylator, and 63 MSS/CIMP-negative. No differences were observed between groups in terms of gender, total number or percentage of patients with polyps, and polyp location. The LS group was younger, had larger polyps size
(1.0 em vs. methylators, $0.4 \mathrm{~cm}, \mathrm{p}=0.02$ ), and more malignant polyps $(4 / 30)$ vs. methylators $(0 / 26)$ and MSS/CIMP-N (1/1 00), $\mathrm{p}=0.01$. Although adenomas were the most prevalent polyp type in all groups, serrated polyps were located more frequently in the right colon in methylators ( 9 of 12) compared to LS patients ( 1 of 5$), p=0.01$. Importantly, the percentage of serrated polyps varied by group with $46 \%(12 / 26)$ incidence in methylators compared to $17 \%(5 / 30)$ in LS $(\mathrm{p}=0.02)$ and $24 \%(24 / 100)$ in MSS/CIMP-negative patients $(\mathrm{p}=0.03)$.

\section{Conclusions}

Polyp burdens associated with MSI-H CRC vary depending on the etiology of microsatellite instability. While LS patients tend to develop adenomas similar to the general population, an increased relative burden of serrated polyps is associated with the methylator phenotype. These findings may help guide investigation into suspected Lynch syndrome.

Published: 25 May 2010

doi:10.1186/1897-4287-8-S1-P12

Cite this article as: Messick et al:: Do precursor polyp burdens help distinguish Lynch versus non-Lynch microsatellite unstable colorectal cancers? Hereditary Cancer in Clinical Practice 2010 8(Suppl 1):P12.

\footnotetext{
* Correspondence: kaladym@ccf.org

The Sanford R. Weiss, M.D. Center for Hereditary Colorectal Neoplasia,

Digestive Disease Institute, Cleveland Clinic, Cleveland, Ohio 44915, USA
} 